\title{
Articular cartilage degradation is prevented by tanshinone IIA through inhibiting apoptosis and the expression of inflammatory cytokines
}

\author{
PEI-TONG JIA ${ }^{1}$, XING-LIN ZHANG ${ }^{1}$, HAI-NING ZUO ${ }^{1}$, XING LU $^{1}$ and LIN LI ${ }^{2}$ \\ ${ }^{1}$ Department of Orthopedics, Yantaishan Hospital, Yantai, Shandong 264001; ${ }^{2}$ Department of \\ Orthopedics, Shandong Jining No. 1 People's Hospital, Jining, Shandong 272011, P.R. China
}

Received August 9, 2016; Accepted June 14, 2017

DOI: $10.3892 / \mathrm{mmr} .2017 .7340$

\begin{abstract}
The present study aimed to investigate the effect of tanshinone IIA on the degradation of articular cartilage in a rat model of osteoarthritis (OA). The OA rat model was established by anterior cruciate ligament transection (ACLT) and medial meniscus resection (MMx). The animals were treated for 28 days with $0.25-0.5 \mathrm{mg} / \mathrm{kg}$ doses of tanshinone IIA following ACLT + MMx. The knee joints of the rats in the ACLT + MMx group exhibited marked alterations in articular cartilage histopathology and higher Mankin scores, compared with those in the normal group. Tanshinone IIA treatment at a dose of $0.5 \mathrm{mg} / \mathrm{kg}$ significantly inhibited cartilage degradation and improved Mankin scores in the OA rat model $(\mathrm{P}<0.002)$. Tanshinone IIA treatment completely inhibited the ACLT + MMx-induced accumulation of inflammatory cells and disintegration of synovial lining in the rats. An increase in the dose of tanshinone IIA between 0.25 and $0.5 \mathrm{mg} / \mathrm{kg}$ reduced the proportion of apoptotic chrondrocytes from 41 to $2 \%$ on day 29 . Treatment of the rats in the ACLT + MMx group with $0.5 \mathrm{mg} / \mathrm{kg}$ doses of tanshinone IIA markedly inhibited the expression level of matrix metalloproteinase and increased the expression of tissue inhibitor of metalloproteinase in the rat articular cartilage tissues. Tanshinone IIA treatment significantly reduced the levels of inflammatory cytokines, including interleukin-1 $\beta$, tumor necrosis factor- $\alpha$ and nitric oxide in rat serum samples. The protein expression levels of bone morphogenetic protein and transforming growth factor- $\beta$ were significantly increased by tanshinone IIA in the ACLT + MMx rats. Therefore, tanshinone IIA inhibited articular cartilage degradation through inhibition of apoptosis
\end{abstract}

Correspondence to: Dr Lin Li, Department of Orthopedics, Shandong Jining No. 1 People's Hospital, 6 Jiankang Road, Jining, Shandong 272011, P.R. China

E-mail: 1ilindr21@gmail.com

Key words: cartilage, osteoarthritis, cytokines, degradation, apoptosis and expression levels of inflammatory cytokines, offering potential for use in the treatment of OA.

\section{Introduction}

Osteoarthritis (OA) is the most common disorder of joints, which is characterized by alterations in the structure and organization of chondrocytes in the articular cartilage $(1,2)$. The disintegration of cartilage is caused by the disturbance of equilibrium between the formation and degradation of matrix components (3). For the treatment of early stage OA, strategies for the provision of symptomatic relief have been developed, however, the advanced stage can be treated only via surgical intervention (4). The apoptosis of chondrocytes also leads to changes in the biosynthesis of cartilage matrix, which is the primary factor causing the development of the OA (5). Inflammatory processes are associated with the degradation of cartilage and the subsequent development of OA (6). The inflammatory reactions and apoptosis of chondrocytes are initiated by the expression of cytokines $(7,8)$. The apoptosis of chondrocytes is caused by the increased expression of interleukin (IL)-1 $\beta$ through the involvement of matrix metalloproteinases (MMPs) $(9,10)$. The effects of MMPs are regulated by the secreted tissue inhibitors of metalloproteinase (TIMPs). Disturbance of the equilibrium between TIMPs and MMPs is one of the factors involved in the development of OA (11).

The use of anti-inflammatory agents has been a subject of interest for those investigating the treatment of various types of cancer, including breast, colorectal, esophageal, lung and stomach cancer $(12,13)$. Anti-inflammatory compounds are used in clinical practice for the treatment of carcinoma either in the form of herbal medicines or as isolated compounds $(14,15)$. The extract of the roots of Salvia miltiorrhiza Bunge (Danshen), has a long history of traditional medicinal importance in China for the treatment of cardiovascular disorders and hepatitis. Phytochemical investigations of this plant have led to the isolation of certain compounds, including tanshinone I, tanshinone IIA and cryptotanshinone (16). Analysis of these compounds has revealed antibacterial (16), antioxidative (17), anti-inflammatory $(18,19)$ and cytotoxic activities $(20,21)$, and inhibitory effects on platelet aggregation (22). The present study investigated the role of tanshinone II-A in preventing 
the induction of apoptosis and cartilage matrix degradation. Tanshinone II-A was found to inhibit the chondrocyte apoptosis and cartilage matrix degradation induced by anterior cruciate ligament transection (ACLT) and medial meniscus resection $(\mathrm{MMx})$.

\section{Materials and methods}

Animals. Healthy 8-week-old male Sprague-Dawley rats, weighing $180 \mathrm{~g}$, were purchased from the Shanghai Laboratory Animal Commission (Shanghai, China) under license number SCXK 2013-012. The experimental procedures involving animals were performed according to the guidelines for the Care and Use of Laboratory Animals 2010 by the Ministry of Science and Technology of the People's Republic of China. The present study was approved by the ethics committee of Shandong Jimin No. 1 People's Hospital (Shandong, China). Osteoarthritis (OA) was induced in the rats by the methods described by Ying et al (23). Briefly, rats were anesthetized with ether, the right knee was exposed and the patella was dislocated laterally. Subsequently, the right knee was fully flexed, followed by anterior crucial ligament transection and medial meniscus resection using micro-scissors.

Treatment strategy. The rats in the treatments groups were administered intragastrically with $0.25,0.30,0.35,0.40,0.45$ and $0.50 \mathrm{mg} / \mathrm{kg}$ doses of tanshinone IIA for 28 days. The rats in the normal control and ACLT + MMx groups received normal saline for the same duration. The animals were housed under a 12-h light/dark cycle in a humidity-controlled (60-64\%) and sterilized room at $25^{\circ} \mathrm{C}$ with access to fresh water and standard laboratory food ad libitum.

Histological analysis. On day 29 following the completion of treatment, the animals were sacrificed following anesthetization with halothane. The bones (tibia and femur) were removed and then subjected to decalcification using EDTA. The paraffin-embedded bone was cut into thin sections of $2 \mu \mathrm{m}$, which were de-paraffinized in boiling xylene. The sections were subjected to hematoxylin and eosin staining, followed by histopathological examination using a Mankin scale, in which 0 indicated normal cartilage and 12 indicated full disintegration (24). Changes in the synovial lining were determined using the Image-Pro Plus 6.0 image analysis system (Media Cybernetics, Inc., Rockville, MD, USA).

Terminal deoxynucleotidyl transferase-mediated dUTP nick end-labeling (TUNEL) assay for the detection of apoptotic cells. For the analysis of apoptotic cells in the cartilage sections, the sections were washed with $1 \%$ PBS/BSA and then fixed in $4 \%$ paraformaldehyde for $20 \mathrm{~min}$. The sections were permeabilized using $0.1 \%$ Triton-X 100 for 15 min on ice. A TUNEL assay using fluorescein isothiocyanate (FITC)-conjugated dUTP, and an Apoptosis Detection System kit (Roche Diagnostics GmbH, Mannheim, Germany) were used for the analysis of apoptosis, according to the manufacturer's instructions.

Flow cytometric analysis. The chondrocytes of the rats belonging to the ACLT + MMx and Tanshinone IIA treatment groups were examined for apoptosis using Annexin V binding and propidium iodine (PI) staining, followed by flow cytometry. The cells were washed with ice-cold PBS and double-stained with FITC-conjugated Annexin V protein and PI for $30 \mathrm{~min}$. Subsequently, 488-nm laser flow cytometry coupled to a cell sorter (FACSCalibur; BD Biosciences, San Jose, CA, USA) was used for analysis of the stained cells.

Enzyme-linked immunosorbent assay (ELISA) analysis. For determination of inflammatory cytokines, blood samples (4 ml) were collected from the aorta of the abdominal region of the rats, and serum was separated by centrifugation at $3,000 \mathrm{xg}$ for $10 \mathrm{~min}$ at $4^{\circ} \mathrm{C}$. The levels of IL-1 $\beta$ (cat. no. EK0393), tumor necrosis factor- $\alpha$ (TNF- $\alpha$; cat no. EK0526) and inducible nitric oxide synthase (iNOS; cat no. EK0472) were quantified using commercially available ELISA kits (ScienCell Research Laboratories, Carlsbad, CA, USA).

Western blot analysis. The cartilage of the rats was washed and placed into lysis buffer (Sigma-Aldrich; Merck KGaA, Darmstadt, Germany) supplemented with phenylmethylsulfonyl fluoride and aprotinin for $4 \mathrm{~h}$ at $4^{\circ} \mathrm{C}$. The content of protein was determined using a detergent compatible protein assay kit (Bio-Rad Laboratories, Inc.). Equal amounts of extracted protein samples $(50 \mu \mathrm{g})$ was mixed with $2 \mathrm{X}$ SDS buffer, and were separated on a $10 \%$ polyacrylamide gel by electrophoresis. The proteins were then transferred onto a nitrocellulose membrane (Bio-Rad Laboratories, Inc.) followed by incubation in blocking buffer (PBS with $7.5 \%$ non-fat dry milk, 2\% BSA and $0.1 \%$ Tween-20) for $2.5 \mathrm{~h}$ at $4^{\circ} \mathrm{C}$. The protein expression levels of MMP-13 and TIMP-1 were determined following incubation at $4^{\circ} \mathrm{C}$ overnight with the following primary antibodies: Mouse monoclonal anti-MMP-13 (cat. no. MA5-14247, 1:400 in blocking buffer; Pierce; Thermo Fisher Scientific, Inc., Waltham, MA, USA), mouse monoclonal anti-TIMP-1 (cat. no. MA1-773, 1:500 in blocking buffer; Pierce; Thermo Fisher Scientific, Inc.) and anti- $\beta$-actin (cat. no. 2791, 1:800 in blocking buffer; OriGene Technologies, Inc., Beijing, China). The membranes were washed with PBS and $0.1 \%$ Tween-20, followed by incubation with secondary horseradish peroxidase-conjugated goat polyclonal anti-rabbit antibodies (cat. no. 31460, 1:10,000 dilution in blocking buffer; Invitrogen; Thermo Fisher Scientific, Inc.) for $1 \mathrm{~h}$ at $4^{\circ} \mathrm{C}$. The membranes were then washed in PBS and developed using an enhanced chemiluminescence detection system (GE Healthcare Life Sciences, Uppsala, Sweden).

Statistical analysis. Data are expressed as the mean \pm standard deviation. The experiments were repeated at least three times, with the mean of the results presented. The data were analyzed using one-way analysis of variance followed by Student's t-test using SPSS software version 21.0 (IBM Corp., Armonk, $\mathrm{NY}$, USA). $\mathrm{P}<0.05$ was considered to indicate a statistically significant difference.

\section{Results}

Tanshinone IIA prevents articular cartilage disintegration in rats induced by ACLT + MMx. The knee joints of the rats in the ACLT + MMx group showed marked alterations in articular cartilage histopathology, which included roughness 


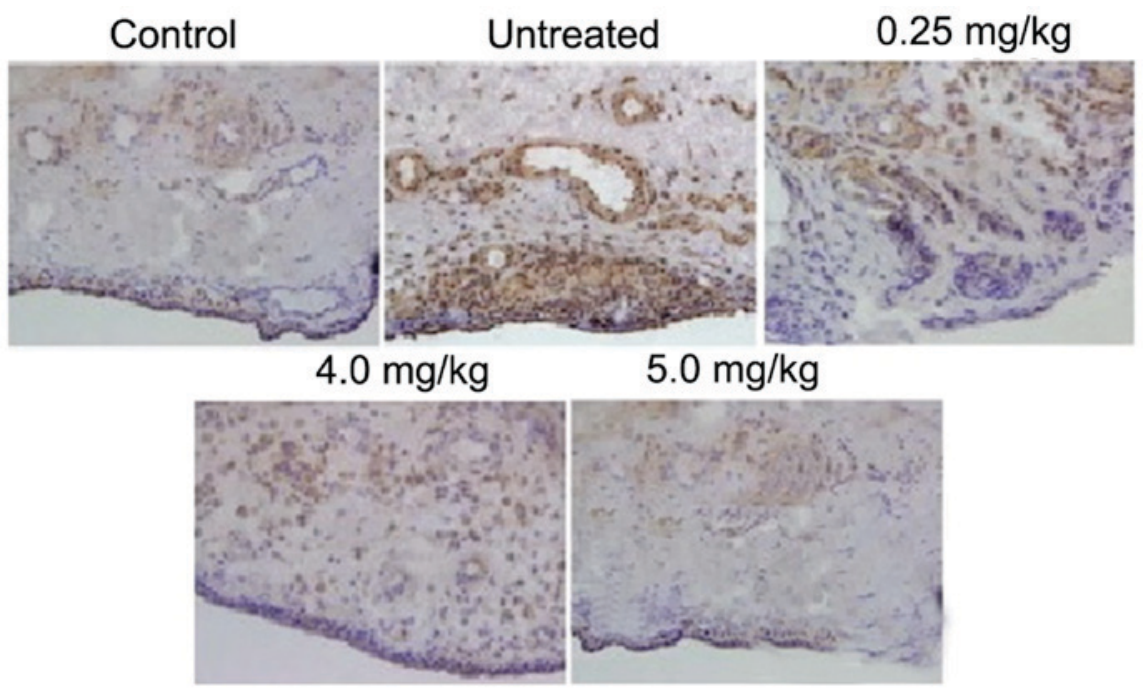

Figure 1. Tanshinone IIA treatment for 28 days inhibited cartilage degradation in the osteoarthritis rat model. The Mankin score was also improved by tanshinone IIA treatment. Histological examination of the cartilage in tanshinone IIA-treated and untreated rats was performed on day 29 post-ACLT + MMx exposure. (magnification, x200). ACLT, anterior cruciate ligament transection; MMx, medial meniscus resection.

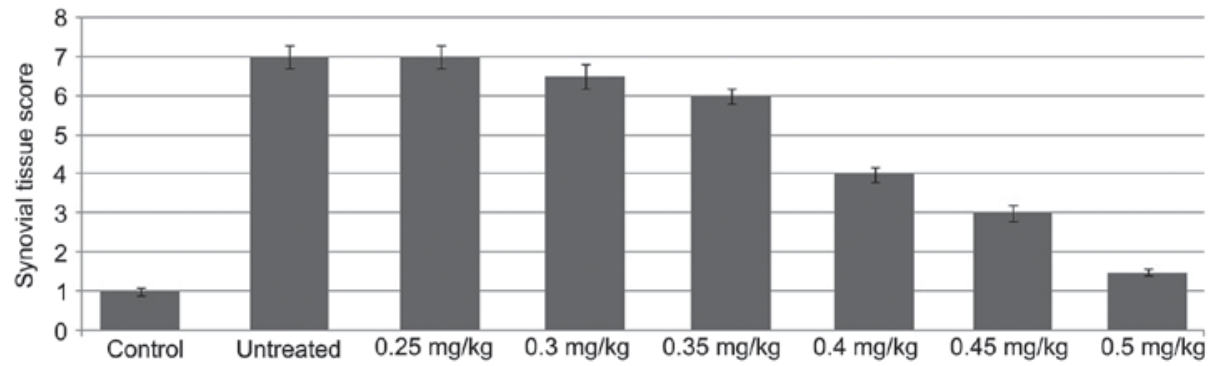

Figure 2. Tanshinone IIA treatment inhibits damage to the synovial lining in the osteoarthritis rat model. Following exposure to ACLT + MMx, the rats were treated with tanshinone IIA for 28 and changes to the synovial lining were analyzed. ACLT, anterior cruciate ligament transection; MMx, medial meniscus resection.

of the cartilage surface and random distribution of chondrocytes (Fig. 1). However, the knee joints of the rats in the normal group exhibited smooth articular cartilage surface and ordered arrangement of chondrocytes. The Mankin score was significantly higher in the rats of the untreated group, compared with the normal rats. Treatment of the rats with tanshinone IIA followed by exposure to ACLT + MMx prevented degradation of the articular cartilage. An increase in the dose of tanshinone IIA between 0.25 and $0.5 \mathrm{mg} / \mathrm{kg}$ had a significant inhibitory effect on the ACLT + MMx-induced degradation of articular cartilage in the rats. Tanshinone IIA treatment at a dose of $0.5 \mathrm{mg} / \mathrm{kg}$ significantly reduced the Mankin score in the ACLT + MMx rats $(\mathrm{P}<0.002)$.

The rats in the ACLT + MMx group exhibited higher expression levels of inflammatory cells in the tissues of the synovium, compared with the rats in the normal group. The lining of the synovium showed hyperplasia, which was absent in the normal group of rats. Treatment of the rats with tanshinone IIA following exposure to ACLT + MMx exhibited a concentration-dependent inhibitory effect on the accumulation of inflammatory cells and disintegration of the synovial lining (Fig. 2). Tanshinone IIA treatment at a dose of $0.5 \mathrm{mg} / \mathrm{kg}$ completely inhibited the ACLT + MMx-induced accumulation of inflammatory cells and disintegration of the synovial lining in the rats.
Tanshinone IIA inhibits ACLT + MMx-induced apoptosis in chondrocytes. In the rats of the untreated group, the proportion of apoptotic chondrocytes was significantly higher (Fig. 3). The proportion of apoptotic cells increased to 52\% following 28 days of ACLT + MMx. However, tanshinone IIA treatment inhibited the induction of apoptosis in chondrocytes in a concentration-dependent fashion. An increase in the dose of tanshinone IIA between 0.25 and $0.5 \mathrm{mg} / \mathrm{kg}$ reduced the proportion of apoptotic chrondrocytes from 41 to $2 \%$ on day 29 .

Tanshinone IIA exhibits inhibitory effects on the $A C L T+M M x$-induced increased expression of MMP-13 and reduced expression of TIMP-1. ACLT + MMx caused a significant increase in the expression of MMP-13 and reduction in the expression of TIMP-1 in the articular cartilage of the rats (Fig. 4). Tanshinone IIA treatment inhibited the ACLT + MMx-induced increased expression of MMP-13 and decreased expression of TIMP-1 in a dose-dependent manner.

Tanshinone IIA inhibits the production of IL-1 $\beta, T N F-\alpha$, and $i N O S$, and promotes the protein expression of BMP and $T G F-\beta$. The serum levels of cytokines involved in inflammatory processes, including TNF- $\alpha$, IL- $1 \beta$ and iNOS, were significantly increased in the ACLT + MMx group. Treatment 

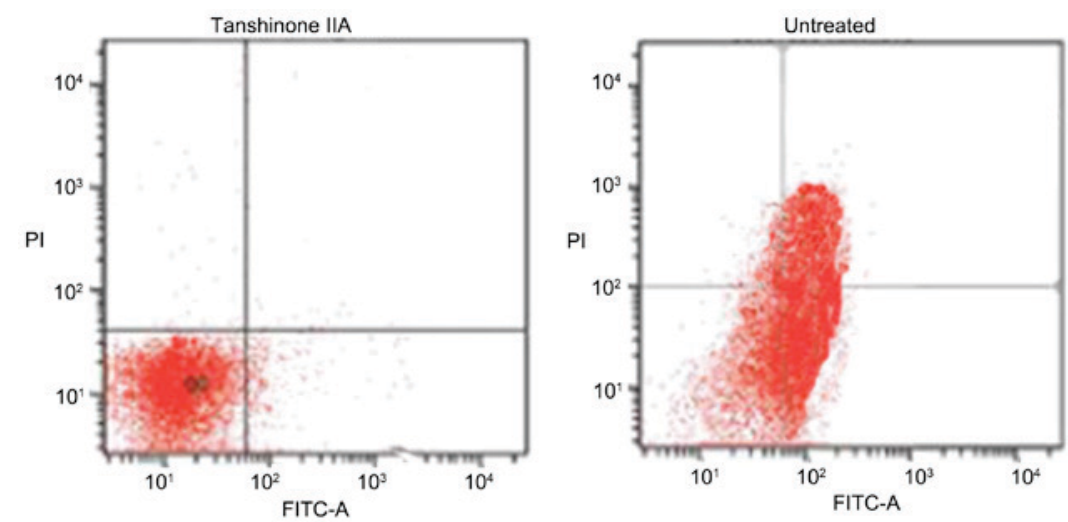

Figure 3. Tanshinone IIA inhibits ACLT + MMx-induced knee joint chondrocyte apoptosis in the rats. The effect of tanshinone IIA on chondrocyte apoptosis was examined following treatment for 28 days. (magnification, x200). ACLT, anterior cruciate ligament transection; MMx, medial meniscus resection; FITC, fluorescein isothiocyanate; PI, propidium iodide.
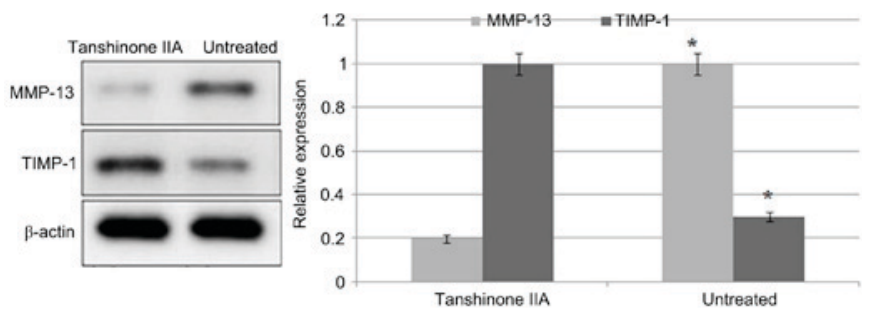

Figure 4. Tanshinone IIA alters the expression levels of TIMP-1 and MMP-13 in the knee joints of the osteoarthritis rat model. The results from the western blot analysis showed significant changes in the expression levels of TIMP-1 and MMP-13 on day 29 post ACLT + MMx exposure. Data are presented as the mean \pm standard deviation. ${ }^{*} \mathrm{P}<0.05$, tanshinone IIA vs. ACLT $+\mathrm{MMx}$ group. ACLT, anterior cruciate ligament transection; MMx, medial meniscus resection; MMP-13, matrix metalloproteinase-13; TIMP-1, tissue inhibitor of metaaloproteinase-1.
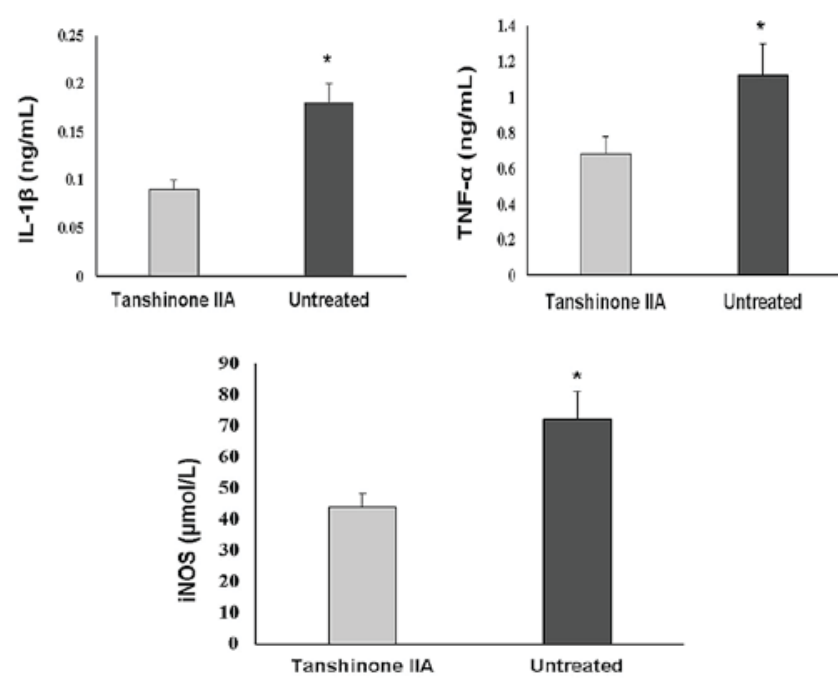

Figure 5. Tanshinone IIA decreased the serum levels of the inflammatory cytokines TNF- $\alpha$, IL- $1 \beta$ and iNOS in ACLT + MMx-treated rats. Data are presented as the mean \pm standard deviation. ${ }^{*} \mathrm{P}<0.05$, Tanshinone IIA vs. ACLT + MMx group. ACLT, anterior cruciate ligament transection; $\mathrm{Mmx}$, medial meniscus resection; TNF- $\alpha$ tumor necrosis factor- $\alpha$; iNOS, inducible nitric oxide synthase; NO, nitric oxide; IL-1 $\beta$, interleukin-1 $\beta$.

with tanshinone IIA at a dose of $0.5 \mathrm{mg} / \mathrm{kg}$ significantly reduced the serum levels of these inflammatory cytokines
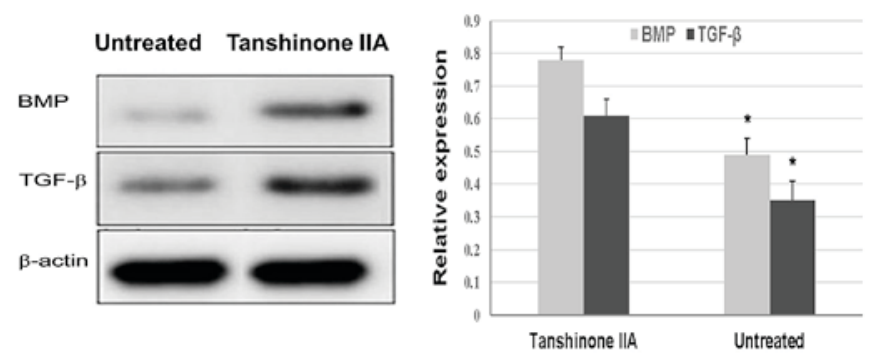

Figure 6. Tanshinone IIA inhibits the protein expression of BMP-2 and TGF- $\beta 1$ in chondrocytes of ACLT + MMx-induced rats. Data are presented as the mean \pm standard deviation. ${ }^{*} \mathrm{P}<0.05$, tanshinone IIA vs. ACLT + MMx group ACLT, anterior cruciate ligament transection; $\mathrm{Mmx}$, medial meniscus resection; BMP-2, bone morphogenetic protein-2; TGF- $\beta 1$, transforming growth factor- $\beta 1$.

(Fig. 5). ACLT + MMx caused a marked decrease in the protein expression of BMP and TGF- $\beta$ in the rat chondrocytes. However, in the rats treated with tanshinone IIA, the protein expression of BMP and TGF- $\beta$ was significantly increased compared with in the untreated group (Fig. 6).

\section{Discussion}

In the development of OA, the apoptosis of chondrocytes and onset of inflammatory processes are vital. Therefore, understanding the mechanism of chondrocyte apoptosis and inflammatory processes, and development of a strategy for their inhibition, offer potential efficient treatment for OA. The present study demonstrated the role of tanshinone IIA in the prevention of OA in the ACLT + MMx rat model. The results revealed that tanshinone IIA efficiently prevented ACLT + MMx-induced degradation of articular cartilage through the inhibition of chondrocyte apoptosis and secretion of inflammatory cytokines.

The apoptosis of chondrocytes degrades articular cartilage, which is the primary factor contributing to the development of the OA. The results obtained in the present study showed that ACLT + MMx induced the apoptosis of chondrocytes in the articular cartilage of the rats, compared with that in normal rats. Treatment of the rats with tanshinone IIA following exposure to ACLT + MMx significantly inhibited the induced apoptosis of chondrocytes. Therefore, tanshinone IIA treatment prevented the damage to the articular cartilage induced by ACLT + MMx 
in rats. Another important factor responsible for the integrity of the cartilage is collagen, and its breakdown has been observed in patients with OA (25). The commonly distributed collagen in cartilage is the type II collagen, and its degradation by MMP-13 is prevented by the intervention of TIMP-1 (26-28). Disturbance of the equilibrium between MMP-13 and TIMPs due to various factors leads to the development of OA (11). The results of the present study revealed that ACLT + MMx increased the expression of MMP-13 in the articular cartilage tissues of the rats. Treatment of the rats with tanshinone IIA following exposure to ACLT + MMx inhibited the increased expression of MMP-13, and maintained the equilibrium between MMP-13 and TIMPs.

Inflammatory cytokines initiate inflammatory reactions, which indicate the beginning of OA. Previous studies have reported that the levels of proinflammatory cytokines, including IL-1 $\beta$, TNF- $\alpha$ and iNOS, were markedly increased in OA patients compared with in healthy controls (29). Furthermore, NO radicals, generated by the activity of iNOS, have been identified as responsible for the induction of cell apoptosis and the increased secretion of MMP-13 in tissues (30). The results of the present study demonstrated that rats of the ACLT + MMx group exhibited increased serum levels of iNOS, IL-1 $\beta$ and TNF- $\alpha$. Conversely, the levels of iNOS IL- $1 \beta$ and TNF- $\alpha$ were significantly decreased following treatment with tanshinone IIA. The biosynthesis of collagen in the cartilage matrix is regulated by the activity of various factors, including TGF- $\beta$ and BMP-2 (31). In the present study, tanshinone IIA treatment in the ACLT + MMx rats increased the expression levels of TGF- $\beta$ and BMP-2.

In conclusion, the present study demonstrated that tanshinone IIA effectively inhibited chondrocyte apoptosis and the degradation of articular cartilage in the ACLT + MMx rat model through inhibiting the expression of inflammatory cytokines. Therefore, tanshinone IIA may be used for the treatment of OA.

\section{References}

1. Gabriel SE, Crowson CS, Campion ME and O'Fallon WM: Direct medical costs unique to people with arthritis. J Rheumatol 24: 719-725, 1997.

2. March LM and Bachmeier CJ: Economics of osteoarthritis: A global perspective. Baillieres Clin Rheumatol 11: 817-834, 1997.

3. Martel-Pelletier J,Lajeunesse D and Pelletier JP:Etiopathogenesis of osteoarthritis. In: Arthritis and Allied conditions: A Textbook of Rheumatology. Koopman WJ and Moreland LW (eds). Vol 2. 15th edition. Lippincott Williams \& Wilkins, Philadelphia, pp2199-2226, 2005.

4. Buckwalter JA and Mankin HJ: Articular cartilage: Degeneration and osteoarthritis, repair, regeneration and transplantation. Instr Course Lect 47: 487-504, 1998.

5. Aigner T and Kim HA: Apoptosis and cellular vitality: Issues in osteoarthritic cartilage degeneration. Arthritis Rheum 46: 1986-1996, 2002.

6. Kapoor M, Martel-Pelletier J, Lajeunesse D, Pelletier JP and Fahmi H: Role of proinflammatory cytokines in the pathophysiology of osteoarthritis. Nat Rev Rheumatol 7: 33-42,2011.

7. Aizawa T, Kon T, Einhorn TA and Gerstenfeld LC: Induction of apoptosis in chondrocytes by tumor necrosis factor-alpha. J Orthop Res 19: 785-796, 2001

8. Fernandes JC, Martel-Pelletier J and Pelletier JP: The role of cytokines in osteoarthritis pathophysiology. Biorheology 39 237-246, 2002.

9. Attur M, Al-Mussawir HE, Patel J, Kitay A, Dave M, Palmer G, Pillinger $\mathrm{MH}$ and Abramson SB: Prostaglandin E2 exerts catabolic effects in osteoarthritis cartilage: Evidence for signaling via the EP4 receptor. J Immunol 181: 5082-5088, 2008.

10. Abramson SB: Osteoarthritis and nitric oxide. Osteoarthritis Cartilage 16 (Suppl 2): S15-S20, 2008.
11. Burger D, Rezzonico R, Li JM, Modoux C, Pierce RA, Welgus HG and Dayer JM: Imbalance between interstitial collagenase and tissue inhibitor of metalloproteinases 1 in synoviocytes and fibroblasts upon direct contact with stimulated T lymphocytes: Involvement of membrane-associated cytokines. Arthritis Rheum 41: 1748-1759, 1998.

12. Harris RE, Namboodiri KK and Farrar WB: Nonsteroidal antiinflammatory drugs and breast cancer. Epidemiology 7: 203-205, 1996.

13. Baron JA and Sandler RS: Nonsteroidal anti-inflammatory drugs and cancer prevention. Annu Rev Med 51: 511-523, 2000.

14. Cohen I, Tagliaferri M and Tripathy D: Traditional Chinese medicine in the treatment of breast cancer. Semin Oncol 29: 563-574, 2002.

15. Kumar NB, Allen K and Bell H: Perioperative herbal supplement use in cancer patients: Potential implications and recommendations for presurgical screening. Cancer Control 12: 149-157, 2005.

16. Lee DS, Lee SH, Noh JG and Hong SD: Antibacterial activities of cryptotanshinone and dihydrotanshinone I from a medicinal herb, Salvia miltiorrhiza Bunge. Biosci Biotechnol Biochem 63: 2236-2239, 1999.

17. Cao EH, Liu XQ, Wang JJ and Xu NF: Effect of natural antioxidant tanshinone II-A on DNA damage by lipid peroxidation in liver cells. Free Radic Biol Med 20: 801-806, 1996.

18. Kang BY, Chung SW, Kim SH, Ryu SY and Kim TS: Inhibition of interleukin-12 and interferon-gamma production in immune cells by tanshinones from Salvia miltiorrhiza. Immunopharmacology 49: 355-361, 2000.

19. Kim SY, Moon TC, Chang HW, Son KH, Kang SS and Kim HP: Effects of tanshinone I isolated from Salvia miltiorrhiza Bunge on arachidonic acid metabolism and in vivo inflammatory responses. Phytother Res 16: 616-620, 2002.

20. Sung HJ, Choi SM, Yoon Y and An KS: Tanshinone IIA, an ingredient of Salvia miltiorrhiza Bunge, induces apoptosis in human leukemia cell lines through the activation of caspase-3. Exp Mol Med 31: 174-178, 1999.

21. Wu WL, Chang WL and Chen CF: Cytotoxic activities of tanshinones against human carcinoma cell lines. Am J Chin Med 19: 207-216, 1991.

22. Wang N, Luo HW, Niwa M and Ji J: A new platelet aggregation inhibitor from Salvia miltiorrhiza. Planta Med 55: 390-391, 1989.

23. Xu Y, Dai GJ, Liu Q, Liu ZL, Song ZQ, Li L, Chen WH and Lin N: Sanmiao formula inhibits chondrocyte apoptosis and cartilage matrix degradation in a rat model of osteoarthritis. Exp Ther Med 8: 1065-1074, 2014.

24. Mankin HJ, Dorfman H, Lippiello L and Zarins A: Biochemical and metabolic abnormalities in articular cartilage from osteo-arthritic human hips. II Correlation of morphology with biochemical and metabolic data. J Bone Joint Surg Am 53: 523-537, 1971.

25. Jubb RW and Fell HB: The breakdown of collagen by chondrocytes. J Pathol 130: 159-167, 1980.

26. Naito K, Watari T, Muta T, Furuhata A, Iwase H, Igarashi M, Kurosawa H, Nagaoka I and Kaneko K: Low-intensity pulsed ultrasound (LIPUS) increases the articular cartilage type II collagen in a rat osteoarthritis model. J Orthop Res 28: 361-369, 2010.

27. Goldring MB, Otero M, Plumb DA, Dragomir C, Favero M, El Hachem K, Hashimoto K, Roach HI, Olivotto E, Borzì RM and Marcu KB: Roles of inflammatory and anabolic cytokines in cartilage metabolism: signals and multiple effectors converge upon MMP-13 regulation in osteoarthritis. Eur Cell Mater 21: 202-220, 2011.

28. Wetzel M, Li L, Harms KM, Roitbak T, Ventura PB, Rosenberg GA, Khokha R and Cunningham LA: Tissue inhibitor of metalloproteinases-3 facilitates Fas-mediated neuronal cell death following mild ischemia. Cell Death Differ 15: 143-151, 2008.

29. Hashimoto S, Nishiyama T, Hayashi S, Fujishiro T, Takebe K, Kanzaki N, Kuroda R and Kurosaka M: Role of p53 in human chondrocyte apoptosis in response to shear strain. Arthritis Rheum 60: 2340-2349, 2009.

30. Del Carlo M Jr and Loeser RF: Nitric oxide-mediated chondrocyte cell death requires the generation of additional reactive oxygen species. Arthritis Rheum 46: 394-403, 2002.

31. Shuler FD, Georgescu HI, Niyibizi C, Studer RK, Mi Z, Johnstone B, Robbins RD and Evans CH: Increased matrix synthesis following adenoviral transfer of a transforming growth factor beta1 gene into articular chondrocytes. J Orthop Res 18: $585-592,2000$ 